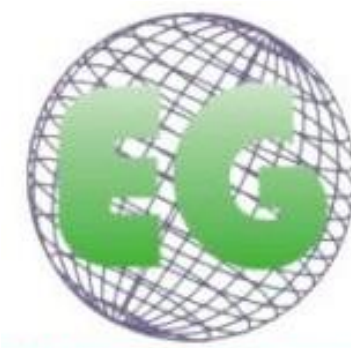

ISSN 1695-6141 $N^{\circ} 48$

\title{
La Gobernanza Enfermera y su impacto en la calidad en la organización sociosanitaria en Catalunya
}

Nursing governance and its impact on the quality of organization of health care services for older people in the Catalan region of Spain

Monserrat Rodó Cobo ${ }^{1}$

${ }^{1}$ Enfermera, Máster en Estudios superiores de enfermería y Máster en Gestión sanitaria. Jefa de Calidad del Hospital Parc Sanitari Pere Virgili. Barcelona. España.

E-mail: rodo.montse@gmail.com

http://dx.doi.org/10.6018/eglobal.16.4.267741

Recibido: $14 / 09 / 2016$

Aceptado: 18/11/2016

\section{RESUMEN:}

Introducción: La Gobernanza enfermera consiste en la toma de decisiones de la líder de enfermería en la organización sanitaria y responde a las necesidades de los grupos de interés. Se estudiaron las líderes enfermeras del ámbito sociosanitario o de atención intermedia de Catalunya (España).

Objetivo: Analizar las variables de Gobernanza en el ámbito sociosanitario y su relación con la calidad percibida por el paciente

Metodología: Se recogieron datos de Gobernanza mediante el Cuestionario de Gobernanza Enfermera (CGE) y Satisfacción de pacientes medidos con Plaensa@ (Encuestas de satisfacción del paciente del CatSalut) 2013. Se obtuvieron los consentimientos y aprobación por el Comité de bioética. Se usaron los programas SPSS de IBM y Atlas- Ti para el análisis de los datos.

Resultados: Respondieron 34 enfermeras $(43,6 \%)$ al CGE y al Plaensa@ 9 de ellas, edad, formación, sexo, años de experiencia y no de camas, de septiembre 2014 a abril 2015. Los datos se analizaron con una significación del $5 \%$ y pruebas estadísticas no paramétricas. Se hallaron correlaciones con la Satisfacción del paciente (Spearman) y: "La calidad influye en las decisiones" $(p=0,006)$, "Investigar y actualizar conocimientos" $(p=0,043)$, y "Desarrollar comunicación estratégica" $(p=0,043)$. Estos resultados se asemejaron a los obtenidos en estudios como el Magnet Modeß日.

Conclusiones: Es conveniente profundizar en la Gobernanza, la Calidad, el Conocimiento y la Comunicación mediante la investigación cualitativa. Para las enfermeras están interrelacionadas entre sí la experiencia en Tomar decisiones, Poder, Representatividad y Cuidados, a pesar de la participación.

Palabras clave: Gobernanza enfermera; Organización sociosanitaria; Calidad

\section{ABSTRACT:}

Introduction: Nursing governance consists of decisions made by nursing leaders in health organizations that respond to the needs of interest groups. Nursing leaders in the realm of social-health or intermediate care in Catalonia (Spain) were studied. 
Objective: Analyze the governance variables in the social-health realm and their relationship with the quality perceived by patients.

Methodology: Governance data was collected through a nursing governance questionnaire and patient satisfaction measured through Plaensa@ (CatSalut 2013 patient satisfaction surveys). The consent of participants and approval by a bioethics committee were obtained. The statistical programs SPSS (from IBM) and Atlas.ti were used for data analysis.

Results: Thirty-four (43.6\%) nurses responded to the nursing governance questionnaire and nine to the Plaensa( ${ }^{\circ}$. Information was collected about age, education, gender, years of experience and number of beds. The data collection took place between September 2014 and April 2015. The data was analyzed with a significance level of $5 \%$ and through nonparametric statistical tests. Correlations were found between patient satisfaction (Spearman) and: "Quality influences decisions" $(p=0.006)$, "Research and update knowledge" $(p=0.043)$, and "Develop strategic communication" $(p=0.043)$. These results were similar to those obtained in studies such as Magnet Model®.

Conclusions: It would be worth delving deeper into the topics of governance, quality, knowledge, and communication through qualitative research. In the view of nurses, decision-making experience, power, representativeness, and care are inter-related, despite participation.

Key words: Nursing governance; Organization of health care services; Quality.

\section{INTRODUCCIÓN}

El modelo asistencial catalán dispone de un nivel de atención llamado sociosanitario o intermedio, que ofrece cuidados multidisciplinarios intensos y expertos de hospitalización que no precisan la tecnología sanitaria hospitalaria y que tampoco se pueden atender en la comunidad por ser complejos, algunos de ellos. Este sector es complementario al hospitalario y a la atención primaria. Se ubica en estructuras hospitalarias de agudos, centros de salud mental, centros para ancianos 0 exclusivamente sociosanitarios. La profesión enfermera, su liderazgo y desarrollo, son claves para la calidad asistencial y la sostenibilidad del sistema sanitario ${ }^{1-3}$.

Hasta el momento poco o nada se sabe sobre el desarrollo de la profesión desde esta perspectiva en nuestro contexto. Sí se ha estudiado en entornos hospitalarios norteamericanos, donde más y durante más años. A este fenómeno se le llama Gobernanza enfermera corporativa o colaborativa. Esta consiste en el desarrollo equilibrado de unas dimensiones que, en manos de la líder de enfermería de la organización sociosanitaria, interactúan y se complementan entre sí y son el Estilo de liderazgo, la Cultura transformacional, la Trasparencia y rendimiento de cuentas, el Poder y autoridad, el Compromiso y sostenibilidad, la Ética y el Equipo multidisciplinar, para satisfacer las necesidades que los grupos de interés tienen sobre ella, los de dentro de la organización y los de fuera de ella ${ }^{4-6}$. Los grupos de interés de la líder enfermera son los Pacientes, las Enfermeras, la Autoridad sanitaria y aseguradora, los Proveedores, las Universidades, las Sociedades científicas, la Dirección y el gobierno de la organización.

Se trata de que las decisiones que toma la enfermera líder, combinen inclusivamente las necesidades de los grupos de interés con la misión y los valores de su organización, de manera que aporte la mayor calidad de decisión posible, autónoma y competencialmente. El ejercicio de Gobernanza, hasta ahora desconocido en el ámbito sociosanitario, podría tener relación con la calidad percibida por el paciente ${ }^{7-11}$, en Catalunya medida con Plaensa $\odot$, Plan de encuestas para la detección de la Satisfacción percibida por el paciente durante su estancia en el centro a cargo del Servei Català de la Salut de la edición del año 2013.

Se analizaron los datos de Gobernanza de las enfermeras líderes del ámbito sociosanitario en Catalunya, y se afirmará o no la relación con la calidad percibida por 
el paciente. La bibliografía consultada refuerza la relación entre Gobernanza y Calidad: según el programa norteamericano Magnet Mode®, implantado durante más de 20 años en el sector hospitalario, cuya implantación y evaluación están orientadas, entre otros, a proporcionar calidad. Tales garantías se relacionan con la cultura de la organización, sostenibilidad, rendimiento enfermero, y bienestar de pacientes y profesionales $^{12-16}$.

\section{MATERIAL Y MÉTODOS}

Para el estudio se invitó a participar a todas las enfermeras con responsabilidad, cargo intermedio o directivo, de los centros sociosanitarios 0 con camas sociosanitarias de Catalunya. Según la lista de centros publicada en la web del CatSalut del año 2014, se contactó telefónicamente con ellas. La investigadora se presentó personalmente, presentó el estudio e invitó a la participación. Se explicitó la confidencialidad y la aprobación por parte del Comité de Bioética de la Universitat de Barcelona. Se explicaron las formas y contacto para disponer del cuestionario, al que dedicarían 15 minutos.

Se llevó a cabo un estudio analítico de las variables y conocimiento sobre ellas. Se optó por la metodología cuantitativa complementada con la obtención de datos narrativos que enriquecieran las respuestas:

- Gobernanza: mediante el cuestionario expresamente diseñado y con validación de contenido por expertos (CGE).

- Sociodemográficas- Nivel de Formación- Estructural o № de camas.

- Calidad percibida: mediante la encuesta de satisfacción Plaensa@ ${ }^{16}$.

EI CGE es un cuestionario autoadministrado de 26 ítems fruto de la revisión de la literatura referida a la toma de decisiones y el modelo de Gobernanza enfermera medido con el Index Professional Nursing Governance (IPNG). El cuestionario IPNG ha sido ampliamente usado en el ámbito hospitalario norteamericano. Cuenta con 88 ítems a responder según el nivel jerárquico que ocupa la enfermera y pregunta por los principios de la Gobernanza compartida mediante seis dimensiones: control de los profesionales, participación, resolución de conflictos, estructuras facilitadoras, accesos a la información y alineamiento, a través de las cuales mide la toma de decisiones para el control de su práctica habitual. Cuenta con las propiedades psicométricas ampliamente evaluadas mediante altos niveles de participación y numerosas ediciones, grupos focales para su discusión así como validación de contenido y consistencia interna necesarios. Hess en diferentes ediciones, evaluó el instrumento e incorporó mejoras hasta que fue aceptado como instrumento de evaluación habitual en los hospitales llamados magnéticos ${ }^{4}$.

Los ítems del CGE se redactaron sobre una acción concreta, en sentido afirmativo, claro y conciso, y se agruparon por dimensiones según su significado tal como la literatura referenció. A su vez, se recogieron datos sociodemográficos (sexo y edad), nivel de formación (grado/ posgrado o máster), mínimo 5 años de experiencia en el mismo centro y número de camas. Además, todas las preguntas del CGE disponían de la opción de redactar texto libre en base a la experiencia u opinión de la líder enfermera encuestada ${ }^{17-24}$.

Este cuestionario fue evaluado por un panel de expertos de 5 profesionales, enfermeras y médicos de los ámbitos de la docencia, la investigación y la gestión, 
mediante el método Delphi. Se pretendió un acuerdo mínimo del $80 \%$ en relevancia y claridad para cada uno de los ítems, a la vez que las posibles respuestas ${ }^{21}$. En la primera ronda de consenso hubo sugerencias de redactado y agrupación de ítems por coincidencia en las ideas principales que se incorporaron a la segunda versión de la encuesta. En la segunda ronda hubo un consenso prácticamente del $100 \%$ tanto en relevancia como en claridad de las preguntas.

Opcionalmente, las enfermeras encuestadas podían aportar los datos que obtuvieron en la edición 2013 de Plaensa(C). Este cuestionario es un instrumento ampliamente usado por la aseguradora sanitaria pública para conocer la satisfacción del paciente en todas las líneas de servicios contratadas por esta desde hace más de 10 años, incluida la sociosanitaria. Su construcción y revisión periódica siguen un estricto y exhaustivo proceso de revisión bibliográfica, grupos de expertos y grupos focales con pacientes. Además todos sus ítems tienen una carga factorial estadísticamente significativa y son discriminantes entre sí ${ }^{16}$.

De los 28 ítems que tiene esta encuesta se seleccionaron 13, que son los relacionados con la enfermería y los cuidados. Interrogan sobre el rol de la enfermera, relación con el paciente y los cuidados que presta el equipo de enfermería. Es con estos ítems con los que se buscó observar la posible relación entre la toma de decisiones y la calidad percibida por el paciente. Asimismo, esta selección de ítems fue validada por el panel de expertos. Las respuestas que solicita este cuestionario recogen categorías que van de muy insatisfecho a muy satisfecho. Excepto la Satisfacción global que se mide con una escala del 1 al 10, donde 1 es muy mala y 10 es excelente.

Las opciones de respuesta, con validación por el panel de expertos, del CGE fueron tipo Likert con cinco categorías: 1- No tengo opinión formada, 2- No estoy de acuerdo y no aplico normalmente, 3- No estoy de acuerdo, pero sí aplico normalmente, 4Estoy de acuerdo pero no aplico normalmente, 5- Estoy de acuerdo y aplico normalmente. De las que la encuestada debía escoger una y complementar su respuesta mediante el texto libre para cada ítem.

La población de estudio fue de 78 enfermeras que cumplieron con los criterios de inclusión: con responsabilidad directiva o intermedia y más de 5 años de experiencia. La recogida y almacén de las respuestas tuvo lugar desde el 2 septiembre de 2014 hasta el 1 de abril 2015, en una base de datos anonimizada.

Para el análisis de las variables de la Gobernanza, Sociodemográficas y del Plaensa@ se llevó a cabo el análisis estadístico bivariante y se seleccionaron las pruebas paramétricas en función del supuesto de normalidad. Se escogieron las pruebas no paramétricas idóneas para tamaños de muestra pequeños: Prueba de $U$ de MannWhitney, Test exacto de Fischer y Coeficiente de correlación de Spearman.

Se aceptó una significación estadística del $5 \%$ ( $p$ valor $=0.05)$, con excepción de un caso justificado en el que se aceptó el 7\% (p valor=0.07). Para el análisis de los datos se usó el programa estadístico SPSS de IBM versión 22.

Para el análisis de los textos obtenidos en el redactado opcional, se usó el método del análisis de contenido con la lectura repetida de las respuestas. La lectura reiterativa y sistemática permitió categorizar las unidades de sentido. Asimismo, se usó el soporte técnico del programa Atlas- Ti versión 5.0. 
Se solicitaron los consentimientos a la participación por parte de la enfermera y del responsable del centro. Se obtuvieron el $100 \%$ de los consentimientos.

\section{RESULTADOS}

Participaron 34 enfermeras $(43.6 \%)$ de centros con contratación por el CatSalut. De estas, $28(82.4 \%)$ eran mujeres, con una media de edad de 46 años (desviación típica de 8.3). En cuanto a la experiencia, recodificada en más o menos de 10 años, 21 (61.8\%) tenían más de 10 años de experiencia y 13 (38.2\%) tenían máximo 10 años de experiencia.

En relación a la distribución de los años de experiencia recodificados (menos o más de 10 años) y la edad (recodificada en menores o mayores de 43 años), se observó que las que tenían hasta 10 años de experiencia 4 (25\%), eran mayores de 43 años y $9(75 \%)$ eran menores de 43 años. Mientras que las que tenían más de 10 años de experiencia 12 (75\%) eran mayores de 43 años, y 9 (50\%) eran menores de 43 años.

Se detectó correlación estadísticamente significativa entre el Sexo (ser mujer) y 4 de los ítems de Gobernanza enfermera (Tabla I): Me aseguro de disponer de procedimientos, protocolos y guías (Mann Whitney, $p$ valor $=0.047$ ), Planifico y desarrollo la gestión de acuerdo con el plan estratégico (Test Fischer $p$ valor $=0.027$, y Mann Whitney $p$ valor $=0.05)$, Conozco los sistemas de gestión de compras y almacén de material sanitario (Mann Whitney, $p$ valor $=0.035$ ), Tengo los conocimientos para influir en las áreas no asistenciales (Fischer, $p$ valor $=0.05$ ). Asimismo, se detectó correlación estadísticamente significativa entre los Años de experiencia recodificados (10 años o menos, o más de 10 años) y el ítem "Investigo y actualizo mis conocimientos mediante la literatura especifica de gestión, dirección y administración sanitaria" (Mann-Whitney, $p$ valor $=0,07$ ).

Se omiten los resultados sobre la Edad y Edad recodificada $(<43,>=43$ años), Nivel de formación y № de camas $(<=50,>50)$, en relación con los 26 ítems por tener un $p$ valor superior a 0.05 . 
Tabla 1. Resultados estadísticamente significativos obtenidos del análisis bivariante entre las variables Gobernanza y el Sexo ( $p$ valor $<=0.05$ ) y Años de experiencia recodificados ( $p$ valor $=0.07$ ).

\begin{tabular}{|c|c|c|}
\hline Ítems del Cuestionario de Gobernanza Enfermera & $\begin{array}{l}\text { Género } \\
\text { (Hombre } \\
\text { o Mujer) }\end{array}$ & $\begin{array}{l}\text { Años de } \\
\text { experiencia } \\
\text { recodificados }\end{array}$ \\
\hline $\begin{array}{l}\text { 1- Adecúo la oferta de servicios a las necesidades de } \\
\text { mis grupos de interés }\end{array}$ & $>0,05$ & $>0,07$ \\
\hline $\begin{array}{l}\text { 2- Incorporo la voz del cliente como fuente de } \\
\text { información }\end{array}$ & $>0,05$ & $>0,07$ \\
\hline $\begin{array}{l}\text { 3- Aseguro la continuidad asistencial del paciente al } \\
\text { alta }\end{array}$ & $>0,05$ & $>0,07$ \\
\hline $\begin{array}{l}\text { 4- Me aseguro que están disponibles procedimientos, } \\
\text { protocolos y guías }\end{array}$ & $=0,047^{*}$ & $>0,07$ \\
\hline $\begin{array}{l}\text { 5- Promuevo la actualización de los sistemas } \\
\text { tecnológicos y de gestión }\end{array}$ & $>0,05$ & $>0,07$ \\
\hline 6- Promuevo el desarrollo de roles y competencias & $>0,05$ & $>0,07$ \\
\hline $\begin{array}{l}\text { 7- Conozco los beneficios y potencialidades de los } \\
\text { modelos sanitarios }\end{array}$ & $>0,05$ & $>0,07$ \\
\hline $\begin{array}{l}\text { 8- Participo en decisiones como las retribuciones } \\
\text { económicas y otras formas de motivación }\end{array}$ & $>0,05$ & $>0,07$ \\
\hline $\begin{array}{l}\text { 9- Participo de forma activa en los procesos generales } \\
\text { de la organización }\end{array}$ & $>0,05$ & $>0,07$ \\
\hline $\begin{array}{l}\text { 10- Influyo en las decisiones del órgano de gobierno de } \\
\text { mi organización }\end{array}$ & $>0,05$ & $>0,07$ \\
\hline $\begin{array}{l}\text { 11- Marco políticas específicas para la identificación y } \\
\text { retención de las enfermeras con talento }\end{array}$ & $>0,05$ & $>0,07$ \\
\hline $\begin{array}{l}\text { 12- Las políticas sanitarias, sociales y económicas, } \\
\text { condicionan mi toma de decisiones }\end{array}$ & $>0,05$ & $>0,07$ \\
\hline $\begin{array}{l}\text { 13- Planifico, desarrollo y evalúo la gestión de acuerdo } \\
\text { con el plan estratégico }\end{array}$ & $=0,05^{*}$ & $>0,07$ \\
\hline $\begin{array}{l}\text { 14- Hago evaluación continua de procesos y resultados } \\
\text { para orientar los cambios }\end{array}$ & $>0,05$ & $>0,07$ \\
\hline $\begin{array}{l}\text { 15- Controlo los costes de la práctica asistencial en } \\
\text { términos generales y analíticos }\end{array}$ & $>0,05$ & $>0,07$ \\
\hline $\begin{array}{l}\text { 16- Los resultados en calidad técnica, percibida y total } \\
\text { propios y externos, influyen mi toma de decisiones }\end{array}$ & $>0,05$ & $>0,07$ \\
\hline $\begin{array}{l}\text { 17- Investigo y actualizo mis conocimientos mediante la } \\
\text { literatura específica }\end{array}$ & $>0,05$ & $=0,07^{*}$ \\
\hline $\begin{array}{l}\text { 18- Participo en iniciativas de benchmarck promovidas } \\
\text { por la aseguradora y la autoridad sanitaria }\end{array}$ & $>0,05$ & $>0,07$ \\
\hline $\begin{array}{l}\text { 19- Establezco convenios de colaboración con } \\
\text { universidades, escuelas de enfermería y auxiliares }\end{array}$ & $>0,05$ & $>0,07$ \\
\hline $\begin{array}{l}\text { 20- Rindo cuentas de forma periódica al órgano de } \\
\text { dirección y al equipo de enfermeras }\end{array}$ & $>0,05$ & $>0,07$ \\
\hline $\begin{array}{l}\text { 21- Desarrollo técnicas de comunicación como } \\
\text { elemento de gestión estratégica }\end{array}$ & $>0,05$ & $>0,07$ \\
\hline $\begin{array}{l}\text { 22- La ética y la legalidad enmarcan la práctica y } \\
\text { cualquier decisión }\end{array}$ & $>0,05$ & $>0,07$ \\
\hline $\begin{array}{l}\text { 23-El modelo de liderazgo es el que requiere la } \\
\text { organización }\end{array}$ & $>0,05$ & $>0,07$ \\
\hline $\begin{array}{l}\text { 24- Conozco los sistemas de gestión de compras y } \\
\text { almacenaje de materiales sanitarios }\end{array}$ & $=0,035^{\star}$ & $>0,07$ \\
\hline $\begin{array}{l}\text { 25- Domino la gestión de las personas en el marco legal } \\
\text { y también personal }\end{array}$ & $>0,05$ & $>0,07$ \\
\hline $\begin{array}{l}\text { 26- Tengo los conocimientos necesarios para influir en } \\
\text { las decisiones clave de las áreas no asistenciales }\end{array}$ & $=0,05^{*}$ & $>0,07$ \\
\hline
\end{tabular}

Respondieron a los ítems de Plaensa@ 15 de las 34 enfermeras participantes. Analizadas sus respuestas, solamente se obtuvieron correlaciones estadísticamente significativas entre 3 de los ítems de Gobernanza enfermera y el ítem de Plaensa@ "Satisfacción global del usuario", analizadas con el coeficiente de Spearman: 
- Los resultados en calidad técnica, percibida y total propios (de la misma organización) y externos, influyen mi toma de decisiones ( $p$ valor $=0.006$ ).

- Investigo y actualizo mis conocimientos mediante la literatura específica ( $p$ valor $=0.043$ ).

- Desarrollo técnicas de comunicación como elemento de gestión estratégica ( $p$ valor $=0.043$ ).

No hubo correlaciones positivas con los ítems de Plaensa@:

- Las enfermeras le escuchan y se hacen cargo de sus preocupaciones.

- El tiempo de espera cuando llama al timbre es aceptable.

- No hablan delante de usted como si no estuviera.

- Sensación de estar en buenas manos.

- Cómo valora la ayuda que le dan.

- Respeto a la intimidad.

- Trato personal de la enfermera.

- Volvería a este hospital en caso de necesitarlo.

En cuanto al análisis de las narraciones recogidas por el CGE, las informantes fueron casi todas mujeres $(n=28)$. Los comentarios fueron 178 en total y los más frecuentes e interesantes se codificaron como Poder de actuación, y Toma de decisiones Asistenciales, por un lado, y de Gobernanza, por otro.

En cuanto al Poder de actuación y la Toma de decisiones Asistenciales, hablaron de la gobernanza del equipo de trabajo: ("sí que se evalúan competencias pero no siempre se tienen los medios para retener a las enfermeras con talento"), ("no dispongo de plena capacidad de gestión del colectivo cuidador (subordinación con gerencia y dirección médica)), ("No contamos con herramientas ágiles de monitorización de indicadores de la actividad enfermera"), su Formación ("Mediante cursos de gestión, másters"), ("si es posible, vía Moodle"), ("Fomento la asistencia a cursos y participación en foros y comisiones") y del Cuidado de pacientes ("Pensamos en las necesidades de los pacientes, (...) e intentamos en la medida de lo posible, responder a ellas. Pero no somos proactivos. No le preguntamos al paciente lo que espera de nosotros, esperamos a que se queje").

Respecto al Poder de actuación y la Toma de decisiones de Gobernanza, relataron elementos de Representatividad ("Participo en el Equipo directivo, pero las decisiones están muchas veces ya pactadas en otros ámbitos"), ("Tengo los conocimientos aunque no siempre influyen en las decisiones clave de las áreas no asistenciales"), y de Accesibilidad al órgano de gobierno ("la influencia en el órgano de gobierno es muy poca, a través de una tercera persona"), así como de Alianzas externas ("tenemos colaboración con la universidad, asociaciones científicas y colegios de profesionales, formando parte de delegaciones").

\section{DISCUSIÓN}

En cuanto a la participación, las enfermeras fueron pocas y en consecuencia, los resultados obtenidos deben tomarse como orientativos 0 punto de partida. Consecuencia de la participación, se produce el sesgo de la representatividad y de la participación: se sabe que no hubo representatividad y que participaron las más inclinadas al tema de estudio. 
En cuanto al análisis de resultados y construcción del instrumento CGE, la ausencia previa de estándar de referencia con el que comparar resultados también supuso una limitación. Asimismo, los datos no fueron recogidos con un instrumento con las propiedades psicométricas necesarias. La validez y la fiabilidad del instrumento de medida (CGE) no se llevaron a cabo por razones de tamaño de la población. A pesar de todo ello, se apostó por generar conocimiento y preguntas que dieran luz a la Gobernanza enfermera ${ }^{25}$.

Esta realidad coincide con Anthony en su revisión sobre los modelos de Gobernanza compartida desarrollados durante 25 años ${ }^{26}$. Planteó que la evidencia se limitaba a mostrar experiencias anecdóticas de mejores resultados en aspectos como buenas relaciones de equipo, menos conflictos, mejor satisfacción de las enfermeras, entre otras. Según ella, hacen falta estudios de largo alcance, segundas generaciones de modelos de Gobernanza en las organizaciones que apliquen métodos de análisis como grupos focales en los que intervengan enfermeras, líderes de enfermería, administradores y otros profesionales.

La influencia de la líder enfermera en el órgano de gobierno no estuvo clara en la mayoría de los casos. Cuestión coherente con el poco impacto en la gestión general de la organización que dijeron tener, debido a que no les era reconocida su competencia y capacidad de liderazgo. Sus críticas coincidieron con los trabajos en el ámbito empresarial de Bastons ${ }^{27}$ y Pérez ${ }^{28}$ que afirman que para que una organización tenga éxito, las metas y los logros de quienes lideran, deben ser compartidos y deben priorizar al bienestar de la comunidad.

Otros componentes de la Gobernanza que se observaron pendientes de desarrollo son la preparación formativa y competencias en el lugar de trabajo para enfermeras y auxiliares de enfermería, y la promoción del buen clima laboral, componente elemental de la calidad de vida de profesionales. Estos principios repercuten directamente en la valoración y la percepción que hace el paciente de la atención que recibe y en la calidad de la asistencia. Así es como se evidenció en la revisión llevada a cabo sobre recursos humanos en la organización sanitaria por Kroth ${ }^{29}$ y Akerdorjet $^{30}$.

Las narraciones de las enfermeras contenían exigencias de reconocimiento como profesionales de la salud en el marco de un nuevo modelo sanitario donde los paradigmas ya cambiaron en consonancia con la evolución de la sociedad y de los ciudadanos que requieren atención sanitaria y social. Reclamaron estar a la altura de representar los cuidados en los más altos niveles de planificación sanitaria. El resto de profesionales sanitarios, dijeron las enfermeras, deben acoger esta presencia con normalidad en beneficio de la salud de los ciudadanos, pero para ello las enfermeras deben estar preparadas y tomar posiciones. Según Havens, en su revisión de estudios sobre Gobernanza enfermera de los años 1990 a 1996, evidenció que no había representación de enfermeras en los órganos de decisión y que su presencia racionaliza los costes y garantiza la satisfacción de los pacientes, entre otros beneficios $^{31,32}$.

En cuanto a la comparación de resultados con los ítems de Plaensa@, resultó desconcertante que ninguno de los 9 ítems escogidos por observar la función enfermera específicamente, no presentaran relación con ninguno de los ítems de la Gobernanza enfermera. Sí la mostraron con la Satisfacción global, lo que coincidió con los resultados de uno de los más importantes estudios y más actuales llevados a 
cabo sobre el tema: según el estudio transversal y multicéntrico europeo y norteamericano que lideró Aiken, en el que participaron cerca de 200.00 personas entre pacientes y enfermeras de más de 1.000 hospitales, se relacionó positivamente la satisfacción y la seguridad del paciente con el trabajo enfermero y su compromiso con la calidad ${ }^{13}$.

Respecto a los resultados en calidad, la investigación y la actualización de conocimientos y la sostenibilidad, se puso de manifiesto su elevado alcance e importancia como para tenerlos en cuenta en estudios posteriores. Exploraciones más profundas podrán explicar los motivos por los cuales haya o no relación con los ítems de Plaensa@ más propios de la enfermería. Por el momento, pues, no se pudo refutar o aceptar con seguridad la relación con la Gobernanza enfermera y la calidad percibida medida con Plaensa@. No obstante, se constataron afirmaciones interesantes de explorar.

En un estudio llevado a cabo por Clavelle, Porter y Drenkard en 2013, en 344 organizaciones con estatus magnético en el que compararon nivel de atracción y retención de las enfermeras medido con el IPNG (Index of Professional Nursing Governance) y con el NWI-R (Work Index-Revised), concluyeron que había relación positiva entre la Gobernanza compartida y la atracción y retención medidas con sendos instrumentos. Ponían énfasis en el papel de la líder enfermera para promover el diseño, la implantación, la facilitación de las estructuras y la evaluación del modelo de gobernanza compartida ${ }^{34}$. Algunos de los ítems del CGE coincidieron con algunos elementos de los instrumentos citados: el Acceso a la información, los Recursos que faciliten la práctica, la Participación en la toma de decisiones, el respeto a los Objetivos, la Autonomía, la Relación médico- enfermera y el Soporte organizacional, entre otros.

La comparación entre instrumentos, parte del diseño, contenido y metodología sugirieron coincidencias aunque también diferencias salvables e insalvables: hay una gran distancia entre el mundo catalán y el norteamericano en recursos para la investigación y en entidades académicas, profesionales y científicas que tengan interés y apuesten por estos estudios, diferencias entre los modelos sanitarios, de provisión de servicios, de formación curricular, de niveles y trayectorias de las enfermeras, entre muchos otros aspectos. Además, como evidenció la bibliografía, cada instrumento de medida debe adaptarse según su modelo sanitario y la organización en el que se aplica $26,33,34$.

\section{CONCLUSIONES}

Siguiendo las líneas, coincidentes y diferentes con la literatura, cabrá reformular las dimensiones y los ítems del CGE incluyendo propuestas de indicadores adecuados al alcance de la enfermería, algunos ejemplos son: la docencia (la presencia de contenido sobre la Gobernanza en la formación de grado), la investigación (la búsqueda de principios de eficiencia y efectividad para la toma de las mejores decisiones), la asistencia (la extensión de estándares de referencia relativos a la seguridad del paciente adecuados a las necesidades de los pacientes sociosanitarios) y la administración y gestión (la representatividad en los órganos de política sanitaria para proteger los intereses de los ciudadanos y los profesionales sanitarios) $35,36,38$. 
Por otro lado, la edad de las enfermeras estudiadas (media de 46 años y mediana de 43 años), los años de experiencia recodificados y el nivel de formación, tuvieron interés. La bibliografía sugiere que la regeneración es una cuestión a plantear. No obstante, se debe complementar con reflexiones sobre la formación y la capacitación académica de las enfermeras. La lectura más adecuada sería que hoy día se prioriza una preparación competencial basada en los años de práctica y no basada en el conocimiento. Aunque, según Bamford, para consolidar la excelencia en la organización, las enfermeras deben dominar tanto la práctica o experiencia acumulada como los conocimientos específicos en su área ${ }^{39}$ y para ello hay que dar las herramientas necesarias y exigir las responsabilidades correspondientes.

Complementariamente, en cuanto al género, los resultados obtenidos del CGE relativos a ser Mujer y el compromiso con una metodología de trabajo determinada (asegurarse de tener herramientas para la toma de decisiones y de la coherencia con el marco estratégico) fuertemente significativas a pesar de la muestra, indican una línea de estudio muy sugerente sobre el valor de ser mujer del Feminismo, opuesto al hembrismo, en la organización ${ }^{31-33}$.

Finalmente, la complejidad de la Gobernanza enfermera merece contar con otros métodos y metodologías para saber más: el análisis del contenido de los relatos de las enfermeras, recogidos con las técnicas oportunas, darán luz a elementos de la calidad, los conocimientos y la sostenibilidad del sistema sanitario en los que las enfermeras debemos posicionarnos inexorablemente.

\section{REFERENCIAS}

1-CatSalut. Departament de Sanitat i Seguretat Social. L'atenció sociosanitària a Catalunya Vida als Anys. 2003. [Internet]. Barcelona [citado 11 noviembre 2016]. Disponible en http://www.gencat.cat/salut/botss/pdf/at sociosanit catalunya 2003.pdf 2-Ortún V. El buen gobierno sanitario. [Internet]. Madrid. Economía de la Salud y Gestión Sanitaria. Springer Healthcare Communications Iberica SL; 2009. P. 12- 15. [citado 8 agosto 2014]. Disponible http://www.econ.upf.edu/ ortun/publicacions/BuenGobSanit16pag.pdf.

3- Rodó M, Torres $P$, Tresseras R. Aproximación a la gobernabilidad enfermera en la organización sociosanitaria en Cataluña. Index Enferm. 2014; 23(4):239-243.

4- Hess R. Measuring nursing governance. Nurs Res. 1998; 47(1):35-42.

5- O’May F, Buchan J. Shared governance: a literature review. Int J Nurs Stud. 1999; 36:281-300.

6- Hess R. Slicing and Dicing Shared Governance. In and Around the Numbers. Nurs Admin Q. 2011; 35(3):235-241.

7- Alimehmeti G, Paletta A. Corporate Governance Indexes: the Confounding Effects of Using Different Measures. JAEBR. 2014; 4(1): 64-79.

8- Gnan L, Hinna A, Monteduro F, Scarozza D. Corporate governance and management practices: stakeholder involvement, quality and sustainability tools adoption. Evidence in local public utilities. J Manag Gov [Internet]. 2013 [citado 11 junio 2014]; 17: 907-937. Disponible http://link.springer.com/article/10.1007/s10997-011-9201-6

9- Giltinane CL. Leadership styles and theories. Nurs Stan [Internet]. 2013 [citado 3 julio 2014]; 27(41):35-39. Disponible en: http://link.springer.com/article/10.1007/s10997-011-9201-6. Citado en: PubMed. PMID: 23905259. 
10- Jackson JP, Clements PT, Averill JB, Zimbro K. Patterns of Knowing: Proposing aTheory for Nursing Leadership. Nurs Econ [Internet]. 2009 [citado 15 marzo 2015]; 27(3):149-159. Disponible en: http://www.ncbi.nlm.nih.gov/pubmed/19558075. Citado en: PubMed; PMID: 19558075.

11- Wong C, Cummings G. Authentic leadership: a new theory for nursing or back to basics?. J Health Organ Manag. 2009 [citado 13 junio 2015]; 23(5):522-538. Disponible en: http://www.ncbi.nlm.nih.gov/pubmed/19862880. Citado en: PubMed; PMID: 19862880. DOI: 10.1108/14777260910984014.

12- Hogston R. Quality nursing care: a qualitative enquiry. J Adv Nurs.1995; $21: 116$ 124.

13- Aiken L, Sermeus W, Van den Heede K, Sloane D, Busse R, McKee M, et al. Patient safety, satisfaction, and quality of hospital care: cross sectional surveys of nurses and patients in 12 countries in Europe and the United States. BMJ [Internet]. 2012 [citado 13 febrero 2015]; 344:e1717. Disponible en: http://www.bmi.com/content/344/bmj.e1717.short.

14- Lin CC. Patient Satisfaction With Nursing Care As an Outcome Variable: Dilemmas for Nursing Evaluation Researchers. J Prof Nurs. 1996; 12(4):207-216. PMID: 8755135.

15- Donabedian A. The quality of care: how can it be assessed?. JAMA.1988; 260(12):1743-1748. DOI:10.1001/jama.1988.03410120089033.

16- Aguado H, Cerdà I, Argimon JM, Murillo C, Canela J. Plan de encuestas de satisfacción del CatSalut- PLAENSAC. Estrategias para incorporar la percepción de la calidad de servicio de los ciudadanos en las políticas de salud. Med Clin [Internet]. 2001[citado 29 abril 2015]; 137(2):55-59. Disponible en: http://www.sciencedirect.com/science/article/pii/S0025775311700306

17- Hendrich A, Chow M. Healthcare Leadership Maximizing The Impact of Nursing Care Quality: a Closer Look at the Hospital work Environment and the Nurse's Impact on Patient-Care Quality. [Internet] Georgia (EUA): Georgia Institute of Technologies \& The Center of Institute Design; 2008. [citado 18 febrero 2015]. Disponible en: www.healthdesign.org.

18- Amezcua M, Gálvez A. Los modos de análisis en investigación cualitativa en salud: perspectiva crítica y reflexiones en voz alta. Rev. Esp. Salud Pública [Internet]. 2002 [citado 21 mayo 2015]; 76(5): 423-436. Disponible en: http://www.indexf.com/cuali/ANALISIS.pdf

19- Martín MC. Diseño y validación de cuestionarios. Matronas Prof [Internet]. 2004 [citado 4 agosto 2014]; 5(17):23-29. Disponible en: http://www.federacionmatronas.org/revista/matronas-profesion/sumarios/i/7192/173/diseno-y-validacion-de-

cuestionarios

20- Rattray J, Jones MC. Essential elements of questionnaire design and development. J CLIN NURS [Internet]. 2007 [citado 20 julio 2014]; 16(3):234-243. Disponible en: http://www.ncbi.nlm.nih.gov/pubmed/17239058. Citado en: PubMed; PMID: 17239058.

21- Astigarraga E. El Método Delphi. Universidad de Deusto Facultad de CC.EE. y empresariales. Donostia - San Sebastián [Internet]. San Sebastián: [citado en 8 agosto 2014]. Disponible en: http://www.prospectiva.eu/zaharra/Metodo delphi.pdf

22- Kramer M, Schmalenberg C. Development and Evaluation of Essentials of Magnetism Tool. J Nurs Adm. 2004; 34 (7/8):365-378.

23- Rattray J, Jones MC. Essential elements of questionnaire design and development. J CLIN NURS .2007; (16): 234- 243.

24- Boynton PM, Greenhalgh T. Selecting, designing, and developing your questionnaire. BMJ. 2004; (328); 1312- 15. 
25- Sarrado JJ, Clèries X, Ferrer M, Kronfly E. Evidencia científica en medicina:¿única alternativa?. Gac Sanit. 2004; 18(3):235-244.

26- Anthony MK. Shared Governance Models: The Theory, Practice, and Evidence. Silver Spring. Online Journal of Issues in Nursing [Internet]. 2004 [citado 4 agosto 2014];

9(1).

Disponible

en:

http://www.nursingworld.org/MainMenuCategories/ANAMarketplace/ANAPeriodicals/O JIN/TableofContents/Volume92004/No1Jan04/SharedGovernanceModels.html

27- Bastons M. La Toma de decisiones en la organización. $1^{\text {a }}$ edición. Barcelona: Editorial Ariel; 2000. p. 55- 75.

28- Pérez JA. Fundamentos de la Dirección de Empresas. 6 edición. Madrid: Ediciones Rialp; 2006. p. 135-141.

29- Kroth M, Kealer C. Human Resource Development Review. [Internet]; 8(4). Idaho: SAGE Publications; 13 August 2009. [citado 13 agosto 2014]. Disponible en: http://hrd.sagepub.com/content/8/4/506.full.pdf+html

30- Akerdorjet K, Severinsson E. The state of the science of emotional intelligence related to nursing leadership: an integrative review. J Nurs Manag. 2010; 18:363-382. DOI:10.1111/j.1365-2834.2010.01087.x.

31- Havens DS. An update on nursing involvement in hospital governance: 19901996.Nurs Econ.1998; 16(1):6-11.

32- Anderson R. Future Organizational Leadership. J PROF NURS. 1997:13(6);334.

33- Argandoña A. The Stakeholder Theory and the Common Good. Research Paper. IESE University of Navarra. 1998 [citado 23 febrero 2015]; 335:2-13. Disponible en: http://www.iese.edu/research/pdfs/DI-0355-E.pdf.

34- Clavelle JT, Porter O'Grady T, Drenkard K. Structural Empowerment and the Nursing Practice Environment in Magnet ${ }^{\circledR}$ Organizations. J Nurs Adm. 2013; 43(11):566-573. DOI:10.1097/01.NNA.0000434512.81997.3f.

35- Salvà $A$, Roqué $M$, Vallés $E$, Bustins $E$, Rodó $M$, Sánchez $P$. Descripción del perfil de complejidad de los pacientes admitidos en unidades sociosanitarias de larga estancia entre los años 2003 y 2009. Rev Esp Geriatr Gerentol. 2014; 49(2):59-54. DOI: 10.1016/j.regg.2013.07.006.

36- Welton JM. Business intelligence and nursing administration. J Nurs Adm [Internet]. 2014 [citado 20 abril 2015]; 44(5):245-246. Disponible: https://www.ncbi.nlm.nih.gov/pubmed/24759193. Citado en: PubMed; DOI: 10.1097/NNA.0000000000000060. PMID: 24759193.

37- Manojlovich M. Power and empowerment in nursing: Looking backward to inform the future. Silver Spring. Online Journal of Issues in Nursing [Internet]. 2007 [citado 2 junio 2014]; 12(1). Disponible http://www.nursingworld.org/MainMenuCategories/ANAMarketplace/ANAPeriodicals/O JIN/TableofContents/Volume122007/No1Jan07/LookingBackwardtolnformtheFuture.ht mIWhile

38- Medina JL, Clèries X, Nolla M. El desaprendizaje: propuesta para profesionales de la salud críticos. FEM [Internet]. 2007 [citado 4 marzo 2015]; 10(4):209-216. Disponible en: http://scielo.isciii.es/pdf/edu/v10n4/colaboracion2.pdf

39- Bamford A. Shared governance: A vehicle for engagement and change. Nurse Educ Today. 2012; (32):191-194. DOI 10.1016/j.nedt.2011.12.009.

ISSN 1695-6141

๑ COPYRIGHT Servicio de Publicaciones - Universidad de Murcia 